\title{
A case of primary rhabdomyosarcoma of the breast
}

\author{
Venkata Satya Suresh Attili · Hemant K. Dadhich · Clementeena RamaRao • P. P. Bapsy • \\ C. Ramachandra · G. Anupama
}

Received: March 2006 / Accepted: June 2007

\begin{abstract}
Primary rhabdomyosarcoma of the breast is a rarely reported in adults, and it occurrence is mostly observed in children. We report a case of primary rhabdomyosarcoma of breast in a 40-year-old lady, who presented in early stage and is in complete remission after one year of treatment.
\end{abstract}

Keywords Primary rhabdomyosarcoma $\cdot$ Breast • Embryonal

\section{Introduction}

Rhabdomyosarcoma (RMS), the commonest paediatric soft tissue tumour, rarely occurs in the adult population. It represents less than $3 \%$ of all adult primary soft tissue sarcomas. The breast is an extremely rare site of occurrence. Though RMS metastatic to breast is more commonly reported than primary RMS breast, occasional case records of the latter also exist. However, we could find only one case presenting in an adult aged more than 40 years ${ }^{1}$. We report here one such case, which was managed

V. S. S. Attili ${ }^{1}$ • H. K. Dadhich ${ }^{1}$ • C. RamaRao ${ }^{2}$ P. P. Bapsy ${ }^{1}$

- C. Ramachandra ${ }^{1}$ - G. Anupama • A. V. S. Suresh ${ }^{1}(\square)$

${ }^{1}$ Departments of Medical Oncology

${ }^{2}$ Departments of Pathology

Kidwai Memorial Institute of Oncology,

Bangalore - 560029

Tel: $+91 / 080 / 26565671$

Fax: $+91 / 080$ / 26565671

E-mail: sureshattili@yahoo.com in our institute according to the IRS III (Intergroup Rhabdomyosarcoma Study) protocol.

\section{Case report}

A 40-year-old woman presented to us with 5 months history of a $4 \times 3 \mathrm{~cm}$ lump in the upper external quadrant of the right breast. Clinical examination revealed palpable right axillary nodes. The patient had no other significant medical history. The patient underwent fine needle aspiration cytology of the breast lump and lymph nodes. The cytology from the breast lump suggested sarcomatous tumour. Metastatic work up including computed tomography (CT) of the thorax, abdomen, and pelvis, revealed no evidence of disease elsewhere. Bone scan and bone marrow examination were normal. She underwent modified radical mastectomy. The final histo-pathological report of the breast tumor showed undifferentiated tumour with small round cells suggestive of Rhabdomyosarcoma (embryonal type). This was further confirmed by immuno-histo chemistry (IHC), which was positive for desmin and negative for cytokeratin.

Following a multi-disciplinary meeting, she was subsequently started with 6 courses of combination chemotherapy with cyclophosphamide, vincristine, actinomycin D according to IRS III protocol. The patient continues to be in complete response at the last follow-up done one year post chemotherapy.

\section{Discussion}

In the past, many entities were often loosely referred to as RMS breast, but with the advent of IHC, RMS has been defined to include only that subset of patients who have desmin and myogenin positivity. According to the new 


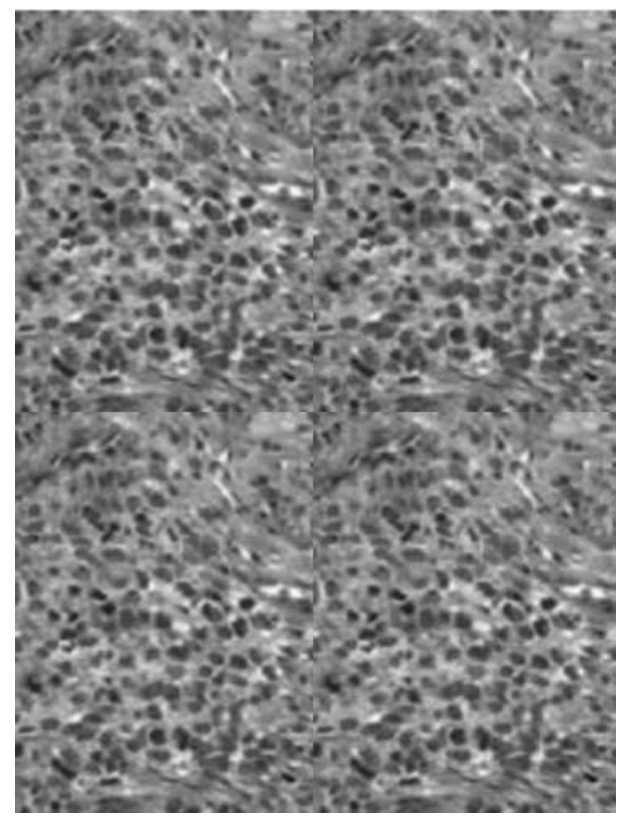

Fig. 1 Microscopy of the breast mass showing undifferentiated tumour with small round cells

definition of RMS of the breast, fewer than 50 cases are reported in the English literature. In one of the largest reported series with a 20 year follow-up period, only 26 cases of either primary (7 cases) or secondary (19 cases) involvement of RMS of breast were noted. The majority of the cases in this series were of alveolar type (24/26). Most of the individual case reports were also of the alveolar histology [2]. All were young females, with ages ranging from 11.5 to 20.2 years. We could only find one IHC proven case of primary RMS of the breast presenting after the fourth decade [1].

These tumors are usually very aggressive, with early and widespread metastases at presentation [3]. In the pediatric population, a few radiographic appearances have been proposed that might differentiate between carcinoma and RMS of the breast. In adolescents, both these entities are known to occur and their differentiation is important. This may be predicted reasonably well by their radiological appearance [4].

Though the survival in most of the cases is not known, Hays et al reported it to be between 3 months to 7 years after diagnosis [2]. As expected, the survival in metastatic RMS is poor as compared to primary RMS of the breast. However, the exact outcome in adults is unknown owing to its rarity.

The present case is unusual in a few aspects

1. Age of presentation in fourth decade, which is extremely uncommon

2. Early stage at presentation

3. Embryonal morphology, which was again rare

\section{References}

1. Antoine Italiano, Rémy Largillier, Isabelle Peyrottes, JeanMichel Hannoun-Levy, Michel Lallement, Antoine Thyss (2005) Primary Embryonal Rhabdomyosarcoma of the Breast in an Adult Female. The Breast Journal 11 (3):214

2. Hays DM, Donaldson SS, Shimada H, Crist WM, Newton WA Jr, et al. (1997) Primary and metastatic rhabdomyosarcoma in the breast: neoplasms of adolescent females, a report from the Intergroup Rhabdomyosarcoma Study. Med Pediatr Oncol 29(3):181-189

3. Vishnevskaia IaV, Sharoev TA, Stepanova EV, Osipova LV. (2004) Rhabdomyosarcoma of the breast in girls. Arkh Patol 6(4):47-51

4. Binokay F, Soyupak SK, Inal M, Celiktas M, Akgul E, Aksungur E. (2003) Primary and metastatic rhabdomyosarcoma in the breast: report of two pediatric cases. Eur J Radiol 48(3): $282-284$ 